\title{
VARIATION OF METRIC TRAITS UNDER SUBDIVISION AND INBREEDING
}

I. BARRAI

World Health Orgonisotion, 1211 Geneva 27, Switzerland

Received 17.xi.71

WRIGHT (1969) reviewed the joint effects of subdivision and inbreeding on the mean and variance of quantitative traits of Mendelian populations. In this paper a simple derivation of these population parameters in the case of complete additivity of gene effects is presented, and a finer resolution of the effects of subdivision and inbreeding on variances is given.

In a population subdivided into isolates, the genotypic structure of the population depends also on the covariances between inbreeding levels and gene frequencies (Barrai, 1971; see also Nei, 1965) so that the array describing the relation between genotype frequencies and a completely additive gene effect might be as in table 1 .

TABLE 1

\begin{tabular}{clc} 
Genotype & \multicolumn{1}{c}{ Frequency $f(X)$} & $\begin{array}{c}\text { Coded } \\
\text { metric trait } X\end{array}$ \\
$A A$ & $p^{2}+p \bar{q} \bar{F}+\sigma_{q}^{2}(1-\bar{F})+\sigma_{q F}-\sigma_{q}{ }^{2} F$ & 2 \\
$A a$ & $2 p \bar{q}-2 p \bar{q} F-2 \sigma_{q}^{2}(1-\bar{F})-2 \sigma_{q} F+2 \sigma_{q}{ }^{2} F$ & 1 \\
$a a$ & $\bar{q}^{2}+p \bar{q} F+\sigma_{q}^{2}(1-\bar{F})+\sigma_{q F}-\sigma_{q}{ }^{2} F$ & 0
\end{tabular}

The mean $\bar{X}$ in the whole population is independent of subdivision and of the local mating system; it is $X=2 p$.

The variance $\sigma_{I T}^{2}$ of the metric trait $X$ in the population is:

$$
\sigma_{I T}^{2}=2 \bar{p} \bar{q}+2 \bar{p} \bar{q} \bar{F}+2 \sigma^{2}(1-\bar{F})+2 \sigma_{q F}-2 \sigma_{q F}^{2} .
$$

In a random mating population, with no subdivisions and no local inbreeding, the variance is:

$$
\sigma_{0}^{2}=2 \bar{p} \bar{q}
$$

It is now convenient to define

$$
\begin{gathered}
\phi=\frac{\sigma_{q}^{2}}{\bar{p} \bar{q}^{\prime}} \\
F_{G M}=\frac{\sigma_{q} F}{\bar{p} \bar{q}}
\end{gathered}
$$

and $F_{N M}=\frac{\sigma_{q F}^{2}}{\bar{p} \bar{q}}$, so that the variance formulation becomes,

$$
\sigma_{I T}^{2}=\sigma_{0}^{2}\left[1+\bar{F}+\phi(1-\bar{F})+F_{G M}-F_{N M}\right] .
$$

It should be noted that the variance is also influenced by the covariances between inbreeding in local isolates (say, in individual plots or strains in controlled breeding experiments) and the gene and genotype frequencies. 


\section{ANAlysis of InBREeding AND sUbdivision EFFects}

When local inbreeding is constant, covariances with $F$ are nil, and

$$
\sigma_{I T}^{2}=\sigma_{0}^{2}[1+F+\phi(1-F)]
$$

and the total variance has four components

$$
\sigma_{I T}^{2}=\sigma_{0}^{2}+\sigma_{0}^{2} F+\sigma_{0}^{2} \phi-\phi F \sigma_{0}^{2} .
$$

If the average local inbreeding is nil, covariances with gene frequencies may nevertheless be different from zero, as for example when there are plots with forced heterozygosis due to artificial fertilisation. Then,

$$
\sigma_{I T}^{2}=\sigma_{0}^{2}\left[1+\phi+F_{G M}-F_{N M}\right]
$$

and the total variance has again four components, the ubiquitous $\sigma_{0}^{2}$ and

$$
\sigma_{0}^{2} \phi+\sigma_{0}^{2} F_{G M}-\sigma_{0}^{2} F_{N M} \text {. }
$$

If random mating is practised within isolates, plots or strains, so that $F$ tend to zero in each, subdivision is the main factor which affects variance:

$$
\sigma_{I T}^{2}=\sigma_{o}(1+\phi) \text {. }
$$

\section{ANALYsis OF VARIANCE}

It may be useful to present a model for the analysis of variance within and between means under local inbreeding and subdivision, as given also by Wright (1969), taking however into consideration variable inbreeding within isolates.

It is convenient to compute first the variance between means as given by $\mathrm{Li}(1955)$, which is:

$$
\sigma_{M}^{2}=4 \sigma_{q}^{2}=2 \sigma_{0}^{2} \phi
$$

so that, by subtraction,

or

$$
\begin{gathered}
\sigma_{W}^{2}=\sigma_{0}^{2}+\sigma_{0}^{2} \bar{F}-\sigma_{0}^{2} \phi-\sigma_{0}^{2} \bar{F} \phi+\sigma_{0}^{2} F_{G M}-\sigma_{0}^{2} F_{N M} \\
\sigma_{W}^{2}=\sigma_{0}^{2}\left[(1+F)(1-\phi)+F_{G M}+F_{N M}\right],
\end{gathered}
$$

so that analysis may be arranged as in table 2 .

TABLE 2

\begin{tabular}{llc} 
Source of variation & \multicolumn{1}{c}{ Variance } & Symbol \\
Total & $\sigma_{0}^{2}\left[1+\bar{F}+\phi(1-\bar{F})+F_{G M}-F_{N M}\right.$ & $\sigma_{I T}^{2}$ \\
between means & $2 \sigma_{0}^{2} \phi$ & $\sigma_{M}^{2}$ \\
within means & $\sigma_{0}^{2}\left[(1+\bar{F})(1-\phi)+F_{G M}-F_{N M}\right]$ & $\sigma_{W}^{2}$
\end{tabular}

From the table, equations may be obtained to estimate $\phi$ or $F$ under appropriate conditions. For example, if $F$ is controlled by a specific breeding system, one obtains immediately

$$
\frac{2 \phi}{(1+\bar{F})(1-\phi)+F_{G M}+F_{N M}}=\frac{\sigma_{M}^{2}}{\sigma_{W}^{2}}
$$




$$
\frac{2 \phi}{1+F+\phi(1-\bar{F})+F_{G M}+F_{N M}}=\frac{\sigma_{M}^{2}}{\sigma_{I T}^{2}},
$$

which reduces, when $F$ is the same in all isolates, to

$$
\phi=\frac{(1+F) \sigma_{M}^{2}}{2 \sigma_{W}^{2}+(1+F) \sigma_{M}^{2}}=\frac{(1+F) \sigma_{M}^{2}}{2 \sigma_{I T}^{2}-(1-F) \sigma_{M}^{2}}
$$

For highly inbred lines in different plots, it is approximately

$$
\phi=\frac{\sigma_{M}^{2}}{\sigma_{I T}^{2}} \text {. }
$$

If one considers that

$$
F_{I T}=F+\phi(1-F)+F_{G M}-F_{N M}
$$

it may be noted that the analysis of variance given in table 2 is identical with the analysis given by Wright.

\section{Summary}

A simple derivation of the variance of completely additive metric traits under subdivision and inbreeding is proposed. The effect of population structure on variances is examined as function of four different components of the inbreeding levels.

\section{REFERENCES}

BARRAI, I. 1971. Subdivision and Inbreeding. Am. F. Hum Genet., 23, 95-96.

LI, c. c. 1955. Population Genetics. The University of California Press.

NEI, M. 1965. Variation and covariation of gene frequencies in subdivided populations. Evolution, 19, 256-258.

WRIGHT, s. 1969. Evolution and the genetics of populations. Vol. II. The University of California Press. 